\title{
On the reciprocal sums of the generalized Fibonacci sequences
}

Han Zhang and Zhengang $\mathrm{Wu}^{*}$

"Correspondence: sky.wzgfff@163.com

Department of Mathematics, Northwest University, Xi'an, Shaanxi, P.R. China

\begin{abstract}
The Fibonacci sequence has been generalized in many ways. One of them is defined by the relation $u_{n}=a u_{n-1}+u_{n-2}$ if $n$ is even, $u_{n}=b u_{n-1}+u_{n-2}$ if $n$ is odd, with initial values $u_{0}=0$ and $u_{1}=1$, where $a$ and $b$ are positive integers. In this paper, we consider the reciprocal sum of $u_{n}$ and then establish some identities relating to $\left\|\left(\sum_{k=n}^{\infty} \frac{1}{u_{k}}\right)^{-1}\right\|$, where $\|x\|$ denotes the nearest integer to $x$.
\end{abstract}

MSC: Primary 11B39

Keywords: generalized Fibonacci sequence; infinite sum; reciprocal sum

\section{Introduction}

For any integer $n \geq 0$, the well-known Fibonacci sequence $F_{n}$ is defined by the secondorder linear recurrence sequence $F_{n+2}=F_{n+1}+F_{n}$, where $F_{0}=0$ and $F_{1}=1$. The Fibonacci sequence has been generalized in many ways, for example, by changing the initial values, by changing the recurrence relation, and so on. Edson and Yayenie [1] defined a further generalized Fibonacci sequence $u_{n}$ depending on two real parameters used in a non-linear recurrence relation, namely,

$$
u_{n}= \begin{cases}a u_{n-1}+u_{n-2} & \text { if } n \text { is even and } n \geq 2, \\ b u_{n-1}+u_{n-2} & \text { if } n \text { is odd and } n \geq 1,\end{cases}
$$

with initial values $u_{0}=0$ and $u_{1}=1$, where $a, b$ are positive integers. This new sequence is actually a family of sequences where each new choice of $a$ and $b$ produces a distinct sequence. When $a=b=1$, we have the classical Fibonacci sequence and when $a=b=2$, we obtain the Pell numbers. Even further, if we set $a=b=k$ for some positive integer $k$, we obtain the $k$-Fibonacci numbers.

Various properties of the Fibonacci numbers and related sequences have been studied by many authors, see [2-4]. Recently, Ohtsuka and Nakamura [5] studied the partial infinite sums of reciprocal Fibonacci numbers and proved that

$$
\left\lfloor\left(\sum_{k=n}^{\infty} \frac{1}{F_{k}}\right)^{-1}\right\rfloor= \begin{cases}F_{n-2} & \text { if } n \text { is even and } n \geq 2, \\ F_{n-2}-1 & \text { if } n \text { is odd and } n \geq 1,\end{cases}
$$

where $\lfloor x\rfloor$ (the floor function) denotes the greatest integer less than or equal to $x$.

Some related works can also be found in [6-13]. In particular, in [13], the authors studied a problem which is a little different from that of [5], namely that of determining the

\section{是 Springer}

๑2013 Zhang and Wu; licensee Springer. This is an Open Access article distributed under the terms of the Creative Commons Attribution License (http://creativecommons.org/licenses/by/2.0), which permits unrestricted use, distribution, and reproduction in any medium, provided the original work is properly cited. 
nearest integer to $\left(\sum_{k=n}^{\infty} \frac{1}{v_{k}}\right)^{-1}$. Specifically, suppose that $\|x\|=\left\lfloor x+\frac{1}{2}\right\rfloor$ (the nearest integer function), and $\left\{v_{n}\right\}_{n \geq 0}$ is an integer sequence satisfying the recurrence formula

$$
v_{n}=a_{1} v_{n-1}+a_{2} v_{n-2}+\cdots+a_{s} v_{n-s} \quad(s \geq 2)
$$

for any positive integer $a_{1}, a_{2}, \ldots, a_{m}$, with the initial conditions $v_{0} \geq 0, v_{k} \in \mathbf{N}, 1 \leq k \leq$ $s-1$. Then, provided $a_{1} \geq a_{2} \geq \cdots \geq a_{m} \geq 1$, we can conclude that there exists a positive integer $n_{0}$ such that

$$
\left\|\left(\sum_{k=n}^{\infty} \frac{1}{v_{k}}\right)^{-1}\right\|=v_{n}-v_{n-1}
$$

for all $n>n_{0}$.

Because the Fibonacci sequence has been generalized to a higher-order recursive sequence, any study on linear recursive sequences has little significance in this context, and we have to consider other non-linear recursive sequences. The main purpose of this paper is concerned with finding expressions for

$$
\left\|\left(\sum_{k=n}^{\infty} \frac{1}{u_{k}}\right)^{-1}\right\|
$$

In fact, this problem is difficult because each item of this sequence relies on the previous relation. In order to resolve the question, we consider the reciprocal sums in two directions: on the one hand to the subsequence $u_{p k+q}$ and on the other to the product form $u_{k} u_{k+2 c+1}$, where $p, q, c$ are non-negative integers and $p \geq 2$. The results are as follows.

Theorem 1 Let $\left\{u_{n}\right\}$ be a second-order sequence defined by (1). For any even $p \geq 2$ and non-negative integer $q<p$, there exists a positive integer $n_{1}$ such that

$$
\left\|\left(\sum_{k=n}^{\infty} \frac{1}{u_{p k+q}}\right)^{-1}\right\|=u_{p n+q}-u_{p n-p+q}
$$

for all $n \geq n_{1}$.

Theorem 2 Let $\left\{u_{n}\right\}$ be a second-order sequence defined by (1). For any integer $c \geq 0$, there exists a positive integer $n_{2}$ such that

$$
\left\|\left(\sum_{k=n}^{\infty} \frac{a^{k} b^{k+2 c+1}}{u_{k} u_{k+2 c+1}}\right)^{-1}-\left(\frac{u_{n} u_{n+2 c+1}}{a^{n} b^{n+2 c+1}}-\frac{u_{n-1} u_{n+2 c}}{a^{n-1} b^{n+2 c}}\right)\right\|=0
$$

for all $n \geq n_{2}$.

Open problem In the light of our investigation, for any positive integer $s \geq 2$ and $l$, whether there exist identities for

$$
\left(\sum_{k=n}^{\infty} \frac{1}{u_{k}^{s}}\right)^{-1} \text { and }\left(\sum_{k=n}^{\infty} \frac{1}{u_{k} u_{k+l}}\right)^{-1}
$$

represent two interesting, albeit challenging, open problems. 


\section{Proofs of the theorems}

We need the following lemma.

Lemma (Generalized Binet's formula) The terms of the generalized Fibonacci sequence $u_{n}$ are given by

$$
u_{n}=\frac{a^{2\left\lfloor\frac{n}{2}\right\rfloor-n+1}}{(a b)^{\left\lfloor\frac{n}{2}\right\rfloor}} \cdot \frac{\alpha^{n}-\beta^{n}}{\alpha-\beta},
$$

where $\alpha=\frac{a b+\sqrt{a^{2} b^{2}+4 a b}}{2}, \beta=\frac{a b-\sqrt{a^{2} b^{2}+4 a b}}{2}$.

Proof See Theorem 2 of [4].

Proof of Theorem 1 From the geometric series as $\epsilon \rightarrow 0$, we have

$$
\frac{1}{1 \pm \epsilon}=1 \mp \epsilon+O\left(\epsilon^{2}\right)=1+O(\epsilon) .
$$

From Lemma and the identity $\alpha \beta=-a b$, we have

$$
u_{p k+q}= \begin{cases}\frac{\alpha^{p k+q}-\beta^{p k+q}}{a^{\frac{p k+-2}{2}} b^{\frac{p k+q}{2}}(\alpha-\beta)} & \text { if } q \text { is even (so that } p k+q \text { is even) } \\ \frac{\alpha^{p k+q}-\beta^{p k+q}}{a b^{\frac{p+q-1}{2}}(\alpha-\beta)} & \text { if } q \text { is odd (so that } p k+q \text { is odd). }\end{cases}
$$

Let

$$
A= \begin{cases}\frac{1}{a^{\frac{p k+q-2}{2}} b^{\frac{p k+q}{2}}(\alpha-\beta)} & \text { if } q \text { is even, } \\ \frac{1}{a b^{\frac{p k+q-1}{2}}(\alpha-\beta)} & \text { if } q \text { is odd. }\end{cases}
$$

Thus,

$$
u_{p k+q}=A \alpha^{p k+q}+O\left(\frac{|\beta|^{\frac{p k}{2}}}{\alpha^{\frac{p k}{2}}}\right) .
$$

Hence,

$$
\begin{aligned}
\frac{1}{u_{p k+q}} & =\frac{1}{A \alpha^{p k+q}\left(1+O\left(\frac{|\beta|^{\frac{p k}{2}}}{\alpha^{\frac{3 p k}{2}}}\right)\right)}=\frac{1}{A \alpha^{p k+q}}\left(1+O\left(\frac{|\beta|^{\frac{p k}{2}}}{\alpha^{\frac{3 p k}{2}}}\right)\right) \\
& =\frac{1}{A \alpha^{p k+q}}+O\left(\frac{|\beta|^{\frac{p k}{2}}}{\alpha^{\frac{5 p k}{2}}}\right) .
\end{aligned}
$$

Thus,

$$
\sum_{k=n}^{\infty} \frac{1}{u_{p k+q}}=\frac{\frac{1}{\alpha^{p n+q}}}{A\left(1-\frac{1}{\alpha^{p}}\right)}+O\left(\frac{|\beta|^{\frac{p n}{2}}}{\alpha^{\frac{5 p n}{2}}}\right)=\frac{\alpha^{p}}{A \alpha^{p n+q}\left(\alpha^{p}-1\right)}\left(1+O\left(\frac{|\beta|^{\frac{p n}{2}}}{\alpha^{\frac{3 p n}{2}}}\right)\right) .
$$


Taking the reciprocal of this expression yields

$$
\begin{aligned}
\left(\sum_{k=n}^{\infty} \frac{1}{u_{p k+q}}\right)^{-1} & =\frac{A \alpha^{p n+q}\left(\alpha^{p}-1\right)}{\alpha^{p}}+O\left(\frac{|\beta|^{\frac{p n}{2}}}{\alpha^{\frac{p n}{2}}}\right) \\
& =A \alpha^{p n+q}-A \alpha^{p n-p+q}+O\left(\frac{|\beta|^{\frac{p n}{2}}}{\alpha^{\frac{p n}{2}}}\right) \\
& =u_{p n+q}-u_{p n-p+q}+O\left(\frac{|\beta|^{\frac{p n}{2}}}{\alpha^{\frac{p n}{2}}}\right) .
\end{aligned}
$$

Therefore, for any even $p \geq 2$ and integer $0<q<p$, there exists $n \geq n_{1}$ sufficiently large such that the modulus of the last error term of identity (2) becomes less than $1 / 2$. This completes the proof of Theorem 1.

Proof of Theorem 2 In the first place, suppose that $k \geq 2$ is even. From Lemma we have

$$
u_{k}=\frac{1}{a^{\frac{k}{2}-1} b^{\frac{k}{2}}} \cdot \frac{\alpha^{k}-\beta^{k}}{\alpha-\beta},
$$

and

$$
u_{k+2 c+1}=\frac{1}{a^{\frac{k}{2}+c} b^{\frac{k}{2}+c}} \cdot \frac{\alpha^{k+2 c+1}-\beta^{k+2 c+1}}{\alpha-\beta} .
$$

The identities $(\alpha-\beta)^{2}=a^{2} b^{2}+4 a b$ and $\alpha \beta=-a b$ now yield

$$
\begin{aligned}
u_{k} u_{k+2 c+1} & =\frac{\alpha^{2 k+2 c+1}+\beta^{2 k+2 c+1}-(\alpha \beta)^{k}\left(\alpha^{2 c+1}+\beta^{2 c+1}\right)}{a^{k+c-1} b^{k+c}(\alpha-\beta)^{2}} \\
& =\frac{\alpha^{2 k+2 c+1}}{\left(a b^{2}+4 b\right)(a b)^{k+c}}+O\left(\left(\frac{\alpha \beta}{a b}\right)^{k}\right) \\
& =\frac{\alpha^{2 k+2 c+1}}{\left(a b^{2}+4 b\right)(a b)^{k+c}}+O(1) .
\end{aligned}
$$

Further, if $k \geq 1$ is odd, the same identity is similarly obtained. Thus, in both cases we have

$$
\begin{aligned}
\frac{1}{u_{k} u_{k+2 c+1}} & =\frac{1}{\frac{\alpha^{2 k+2 c+1}}{\left(a b^{2}+4 b\right)(a b)^{k+c}}+O(1)}=\frac{1}{\frac{\alpha^{2 k+2 c+1}}{\left(a b^{2}+4 b\right)(a b)^{k+c}}\left(1+O\left(\frac{(a b)^{k}}{\alpha^{2 k}}\right)\right)} \\
& =\frac{\left(a b^{2}+4 b\right)(a b)^{k+c}}{\alpha^{2 k+2 c+1}}+O\left(\frac{(a b)^{2 k}}{\alpha^{4 k}}\right) .
\end{aligned}
$$

Hence,

$$
\begin{aligned}
\frac{a^{k} b^{k+2 c+1}}{u_{k} u_{k+2 c+1}} & =\frac{\left(a b^{3}+4 b^{2}\right) a^{2 k+c} b^{2 k+3 c}}{\alpha^{2 k+2 c+1}}+O\left(\frac{(a b)^{3 k}}{\alpha^{4 k}}\right) \\
& =\frac{a^{c+1} b^{3 c+3}+4 a^{c} b^{3 c+2}}{\alpha^{2 c+1}} \cdot \frac{(a b)^{2 k}}{\alpha^{2 k}}+O\left(\frac{(a b)^{3 k}}{\alpha^{4 k}}\right) .
\end{aligned}
$$


Let $B=\frac{a^{c+1} b^{3 c+3}+4 a^{c} b^{3 c+2}}{\alpha^{2 c+1}}$, then

$$
\frac{a^{k} b^{k+2 c+1}}{u_{k} u_{k+2 c+1}}=\frac{B(a b)^{2 k}}{\alpha^{2 k}}+O\left(\frac{(a b)^{3 k}}{\alpha^{4 k}}\right)
$$

Consequently,

$$
\begin{aligned}
\sum_{k=n}^{\infty} \frac{a^{k} b^{k+2 c+1}}{u_{k} u_{k+2 c+1}} & =B \sum_{k=n}^{\infty} \frac{(a b)^{2 k}}{\alpha^{2 k}}+O\left(\sum_{k=n}^{\infty} \frac{(a b)^{3 k}}{\alpha^{4 k}}\right) \\
& =B \cdot \frac{\left(\frac{a b}{\alpha}\right)^{2 n}}{1-\left(\frac{a b}{\alpha}\right)^{2}}+O\left(\frac{(a b)^{3 n}}{\alpha^{4 n}}\right) .
\end{aligned}
$$

Taking the reciprocal of this expression yields

$$
\begin{aligned}
\left(\sum_{k=n}^{\infty} \frac{a^{k} b^{k+2 c+1}}{u_{k} u_{k+2 c+1}}\right)^{-1} & =\frac{1}{B \cdot \frac{\left(\frac{a b}{\alpha}\right)^{2 n}}{1-\left(\frac{a b}{\alpha}\right)^{2}}\left(1+O\left(\frac{|\beta|^{n}}{\alpha^{n}}\right)\right)} \\
& =\frac{1-\left(\frac{a b}{\alpha}\right)^{2}}{B \cdot\left(\frac{a b}{\alpha}\right)^{2 n}}\left(1+O\left(\frac{|\beta|^{n}}{\alpha^{n}}\right)\right) \\
& =\frac{1-\left(\frac{a b}{\alpha}\right)^{2}}{B \cdot\left(\frac{a b}{\alpha}\right)^{2 n}}+O\left(\frac{1}{\alpha^{n}|\beta|^{n}}\right) \\
& =\frac{1}{B} \cdot\left(\frac{\alpha}{a b}\right)^{2 n}-\frac{1}{B} \cdot\left(\frac{\alpha}{a b}\right)^{2 n-2}+O\left(\frac{1}{\alpha^{n}|\beta|^{n}}\right) .
\end{aligned}
$$

On the other hand,

$$
\begin{aligned}
& \frac{u_{n} u_{n+2 c+1}}{a^{n} b^{n+2 c+1}}-\frac{u_{n-1} u_{n+2 c}}{a^{n-1} b^{n+2 c}} \\
& =\frac{\alpha^{2 c+1}}{a^{c+1} b^{3 c+3}+4 a^{c} b^{3 c+2}} \cdot\left(\frac{\alpha}{a b}\right)^{2 n}-\frac{\alpha^{2 c+1}}{a^{c+1} b^{3 c+3}+4 a^{c} b^{3 c+2}} \cdot\left(\frac{\alpha}{a b}\right)^{2 n-2}+O\left(\frac{1}{a^{n} b^{n}}\right) \\
& \quad=\frac{1}{B} \cdot\left(\frac{\alpha}{a b}\right)^{2 n}-\frac{1}{B} \cdot\left(\frac{\alpha}{a b}\right)^{2 n-2}+O\left(\frac{1}{a^{n} b^{n}}\right) .
\end{aligned}
$$

Combining (3) and (4), finally we have

$$
\left(\sum_{k=n}^{\infty} \frac{a^{k} b^{k+2 c+1}}{u_{k} u_{k+2 c+1}}\right)^{-1}-\left(\frac{u_{n} u_{n+2 c+1}}{a^{n} b^{n+2 c+1}}-\frac{u_{n-1} u_{n+2 c}}{a^{n-1} b^{n+2 c}}\right)=O\left(\frac{1}{a^{n} b^{n}}\right)
$$

It follows that for any integer $c \geq 0$, there exists $n \geq n_{2}$ sufficiently large such that the modulus of the last error term of identity (5) becomes less than $1 / 2$. This completes the proof of Theorem 2 . 
Authors' contributions

$\mathrm{HZ}$ obtained the theorems and completed the proof. ZW corrected and improved the final version. Both authors read and approved the final manuscript.

\section{Acknowledgements}

The authors express their gratitude to the referee for very helpful and detailed comments. This work is supported by the N.S.F. (11371291), the S.R.F.D.P. (20136101110014), the N.S.F. (2013JZ001) of Shaanxi Province, and the G.I.C.F. (YZZ12062) of NWU, P.R. China.

Received: 16 October 2013 Accepted: 1 December 2013 Published: 23 Dec 2013

\section{References}

1. Edson, M, Yayenie, O: A new generalization of Fibonacci sequence and extended Binet's formula. Integers 9, 639-654 (2009)

2. Falcón, S, Plaza, Á: On the Fibonacci k-numbers. Chaos Solitons Fractals 32, 1615-1624 (2007)

3. $\mathrm{Ma}, \mathrm{R}$, Zhang, W: Several identities involving the Fibonacci numbers and Lucas numbers. Fibonacci Q. 45, 164-170 (2007)

4. Yi, Y, Zhang, W: Some identities involving the Fibonacci polynomials. Fibonacci Q. 40, 314-318 (2002)

5. Ohtsuka, H, Nakamura, S: On the sum of reciprocal Fibonacci numbers. Fibonacci Q. 46/47, 153-159 (2008/2009)

6. Zhang, W, Wang, T: The infinite sum of reciprocal Pell numbers. Appl. Math. Comput. 218, 6164-6167 (2012)

7. Xu, Z, Wang, T: The infinite sum of the cubes of reciprocal Pell numbers. Adv. Differ. Equ. 2013, Article ID 184 (2013)

8. Wu, Z, Zhang, W: The sums of the reciprocal of Fibonacci polynomials and Lucas polynomials. J. Inequal. Appl. 2012, Article ID 134 (2012)

9. Wu, Z, Zhang, W: Several identities involving Fibonacci polynomials and Lucas polynomials. J. Inequal. Appl. 2013, Article ID 205 (2013)

10. Holliday, S, Komatsu, T: On the sum of reciprocal generalized Fibonacci numbers. Integers 11, 441-455 (2011)

11. Komatsu, T, Laohakosol, V: On the sum of reciprocals of numbers satisfying a recurrence relation of order $\mathrm{s}$. J. Integer Seq. 13, Article ID 10.5.8 (2010)

12. Kilic, E, Arikan, T: More on the infinite sum of reciprocal usual Fibonacci, Pell and higher order recurrences. Appl. Math. Comput. 219, 7783-7788 (2013)

13. Wu, Z, Zhang, H: On the reciprocal sums of higher order sequences. Adv. Differ. Equ. 2013, Article ID 189 (2013)

10.1186/1687-1847-2013-377

Cite this article as: Zhang and Wu: On the reciprocal sums of the generalized Fibonacci sequences. Advances in Difference Equations 2013, 2013:377

\section{Submit your manuscript to a SpringerOpen ${ }^{\ominus}$ journal and benefit from:}

- Convenient online submission

- Rigorous peer review

Immediate publication on acceptance

- Open access: articles freely available online

- High visibility within the field

- Retaining the copyright to your article 